\title{
Criminologie
}

\section{Mutilations corporelles infligées aux femmes : étude victimologique}

\section{H. F. Ellenberger}

Volume 13, numéro 1, 1980

Regards sur la victime

URI : https://id.erudit.org/iderudit/017117ar

DOI : https://doi.org/10.7202/017117ar

Aller au sommaire du numéro

Éditeur(s)

Les Presses de l'Université de Montréal

ISSN

0316-0041 (imprimé)

1492-1367 (numérique)

Découvrir la revue

Citer cet article

Ellenberger, H. F. (1980). Mutilations corporelles infligées aux femmes : étude victimologique. Criminologie, 13(1), 80-93. https://doi.org/10.7202/017117ar d'utilisation que vous pouvez consulter en ligne.

https://apropos.erudit.org/fr/usagers/politique-dutilisation/ 


\section{MUTILATIONS CORPORELLES INFLIGEES AUX FEMMES : ÉTUDE VICTIMOLOGIQUE}

H.F. Ellenberger

Les mutilations et déformations corporelles à caractère traditionnel chez les diverses populations humaines constituent un domaine vaste et encore mal connu qui se situe aux confins de l'ethnologie et de la victimologie. Il en existe une grande diversité, depuis les plus bénignes (colorations, tatouages) jusqu'aux plus graves (perforations, ablations de parties du corps) ${ }^{1}$. Le problème de l'origine et des motivations de telles coutumes est un des plus obscurs de toute l'anthropologie.

Dans cet ensemble disparate, on peut isoler un groupe de mutilations et de déformations qui semblent avoir pour but de victimiser les femmes en portant atteinte au domaine le plus intime de leur personnalité. Il s'agit particulièrement des mutilations sexuelles connues sous le nom impropre de "circoncision féminine ${ }^{2}$. Pour des raisons qui seront exposées plus loin, nous $\mathrm{y}$ ajouterons les mutilations pratiquées naguère en Chine sur les pieds des femmes. Quant à d'autres déformations, comme celles des «femmes girafes » et des «négresses à plateau », elles constituent un autre groupe de déformations qui mériterait une étude à part.

\section{Mutilations sexuelles : (prétendue circoncision féminine)}

On estime que des mutilations sexuelles ont été infligées, à l'heure actuelle, à environ 30 millions de femmes et que des milliers de petites filles en sont victimes tous les jours, principalement dans le nord-est de l'Afrique et dans plusieurs pays d'Afrique noire. On peut classer ces mutilations d'après la gravité de l'atteinte corporelle, d'après leur signification sociologique, et d'après leur répartition géographique.

1. Voir ouvrage d'Adolfo Dembo y J. Imbelloni : Deformaciones intencionales del cuerpo humano de carácter étnico, Buenos Aires, Editorial Nova, s.d. (348 p.).

2. Le présent article était déjà sous presse lorsqu'a paru l'ouvrage de Fran P. Hosken : The Hosken Report: Genital and Sexual Mutilation of Females, Win News, Lexington, Mars 1979. 


\section{Degrés de gravité des mutilations} degrés :

De façon un peu schématique, on peut en distinguer trois $1^{\circ}$ La circoncision simple ou sunna, qui est l'ablation limitée de certaines parties du clitoris.

$2^{\circ}$ La clitoridectomie simple ou élargie, c.-à-d. limitée à la totalité ou presque totalité du clitoris, ou s'étendant aussi sur les parties adjacentes.

$3^{\circ}$ L'infibulation, dite «circoncision pharaonique »: c'est une opération extrêmement grave qui comprend l'ablation du clitoris et des petites lèvres, celle d'une partie importante des grandes lèvres, mutilations suivies par une suture de la vulve effectuée de manière à réduire l'orifice vaginal à un diamètre juste suffisant pour permettre l'écoulement de l'urine et du sang menstruel.

Le terme de «circoncision féminine » est tout à fait impropre. Même quand il s'agit d'une clitoridectomie simple, l'opération ne peut être mise en parallèle avec la circoncision masculine, laquelle ne diminue en rien le plaisir sexuel, à la différence de la clitoridectomie. Quant à l'infibulation, c'est bien plutôt à la castration chez l'homme qu'il faudrait la comparer.

\section{Classification sociologique}

$1^{\circ}$ La forme de mutilation la plus grave, dite «circoncision pharaonique », ne semble pas liée, du moins actuellement, à un rite. Il s'agit simplement d'une coutume qui peut se présenter avec l'apparence inoffensive d'une fête de famille. Mais cette coutume se perpétue par une tradition tenace et elle est entourée d'une barrière de secret, si bien qu'il est le plus souvent difficile d'obtenir des renseignements, même parfois sur son existence. Géographiquement son lieu d'élection est le nord-est de l'Afrique, et tout particulièrement la Somalie.

$2^{\circ}$ Dans nombre de pays d'Afrique noire, l'excision totale ou partielle du clitoris fait partie des rites d'initiation auxquels sont astreintes toutes les fillettes de 10 à 12 ans.

\section{Répartition géographique}

La répartition géographique des mutilations sexuelles féminines est mal connue. Il est certain qu'il existe d'assez grandes 
différences d'une région à l'autre, quant au pourcentage des femmes mutilées, quant à la gravité de la mutilation infligée, et quant à l'âge où elle est pratiquée. L'infibulation a été assez répandue en Égypte et y existe encore, bien qu'elle ait été interdite en 1952. Elle est fréquente en Nubie, en Éthiopie, au Soudan, et surtout en Somalie où pratiquement toutes les femmes la subissent sous sa forme la plus mutilante. La clitoridectomie, simple ou élargie, est un élément des rites d'initiation chez de nombreuses populations noires d'Afrique, jusqu'en Guinée, en Nigéria et au Kenya. Au Yemen et en Arabie du sud, la circoncision féminine existe sous une forme un peu différente : elle y est pratiquée au $7^{\mathrm{e}}$ jour après la naissance. On note qu'elle semble inexistante au Maghreb.

Nous nous limiterons ci-après à un bref exposé de l'infibulation telle qu'elle est encore pratiquée au Soudan et de la clitoridectomie telle qu'elle existe encore au Kenya.

\section{L'infibulation dite \& circoncision pharaonique 》: description}

La forme de mutilation sexuelle la plus grave et la plus dangereuse, l'infibulation, est pratiquée aujourd'hui en Égypte, en Nubie, au Soudan, et surtout en Somalie. Nous suivrons la description qu'en a donnée au Soudan le Dr El Sayed Mirghani ${ }^{\text {s. }}$

La fillette qui va en être la victime ignore tout ce qui va se passer. On la prépare en lui disant qu'elle va subir une purification, qui sera un jour de joie célébré en son honneur; elle recevra des cadeaux et de nouveaux habits, ses mains seront teintes en jaune avec le henné. Tout se passe entre femmes; aucun homme n'y sera admis, pas même le père. L'opératrice est une vieille femme, la gedda (mot qui signifie grand-mère). L'opération, qui dure une vingtaine de minutes, est pratiquée sans anesthésie et sans aucune trace d'asepsie ou d'antisepsie. Elle est atrocement douloureuse, c'est pourquoi, afin d'empêcher l'enfant de se débattre, plusieurs femmes lui maintiennent vigoureusement les bras, les jambes et le bassin, tout en criant à tue-tête pour couvrir les hurlements de l'enfant. Après un semblant de lavage à l'eau, la gedda prend un vieux couteau (souvent sale et mal aiguisé), avec

3. El Sayed Mirghani el Sayed : De la pratique de la clitoridectomie, nymphectomie, vulvectomie et infibulations rituelles chez les petites filles dans certains pays d'Afrique et d'Asie. Thèse méd., Paris, Université Descartes, 1973, no 118. 
lequel elle découpe et enlève le clitoris et une partie des régions adjacentes, fait une incision le long du bord inférieur des grandes lèvres puis rapproche les deux plaies de façon à déterminer une soudure qui recouvrira la vulve en pont jusqu'à la moitié de lorifice vaginal. La plaie est ensuite arrosée à l'eau froide, saupoudrée de poudre de myrrhe, suturée à l'aide des épines de l'acacia nain. L'opérée est ligotée des genoux au ventre et laissée 15 jours dans cette position. La cicatrisation achevée, le périnée deviendra une surface lisse où l'ouverture du vagin ne sera plus qu'un orifice quasi-punctiforme pour l'écoulement de l'urine et des règles. La fillette sera désormais une femme cousue », mais ses tribulations seront loin d'être finies.

En effet, la « circoncision pharaonique » peut être considérée comme une opération en trois temps ; or l'excision et l'infibulation ne constituent que le premier temps. Le deuxième temps est l'\& ouverture » pratiquée la veille du mariage, c'est-à-dire vers l'âge de 12 à 15 ans. La sœur et la mère du fiancé vérifient la qualité de la fermeture de la jeune fille, qui est ensuite livrée au mari. Celui-ci, à l'aide d'un instrument tranchant, élargit de bas en haut l'orifice vaginal, faute de quoi il lui serait impossible d'avoir des relations sexuelles avec la mariée. Les premières relations intimes, en particulier celles qui suivent chacune des défibulations, sont extrêmement douloureuses.

Quant à l'accouchement, on peut le considérer comme étant le troisième temps. On attend que la tête de l'enfant s'engage, alors la gedda pratique une ouverture d'un coup sec. L'accouchement terminé, la plaie est arrosée d'eau bouillante et la femme est ligotée à nouveau pour tout remettre en état, c'est-à-dire que tout est refermé jusqu'à l'accouchement suivant.

\section{L'infibulation : ses suites et ses complications}

Les complications sont fréquentes, mais ici aussi il est extrêmement difficile d'en faire une évaluation statistique, vu l'atmosphère de secret qui entoure cette coutume. On peut distinguer des complications immédiates ou tardives, et des complications physiques ou psychiques.

Parmi les complications immédiates, on cite des cas de choc opératoire parfois mortel, des hémorragies, pouvant, elles aussi, entraîner parfois la mort, des lésions des organes voisins tels 
que la vessie et l'urètre, des symptômes de rétention urinaire, des infections fréquentes et souvent graves : septicémies, tétanos, gangrène, et des infections localisées.

Parmi les complications tardives d'ordre physique, il faut noter la fréquence des infections chroniques des voies urinaires, ainsi que des troubles fonctionnels tels que l'hématocolpos (rétention de sang menstruel par obstruction vaginale). Des arthrites sont fréquentes et apparaissent dès le premier mois après l'opération. D'autres complications se révèlent au moment de la défibulation : cicatrices vicieuses, kystes, fistules vésico-vaginales ou recto-vaginales, prolapsus, cystocèle. Non moins fréquentes sont les complications obstétricales, d'où résultent une forte morbidité et une certaine mortalité. Il ne faut pas sous-estimer les complications pratiques d'ordre médical : impossibilité de pratiquer des examens au spéculum, impossibilité du sondage vésical. Parmi les complications à long terme, on note encore des retards de croissance et de puberté.

Les complications psychiques sont certainement très fréquentes. D'après Lagraulet ${ }^{4}$ ces femmes deviennent insensibles, sans tendresse pour leurs enfants et impassibles devant leurs souffrances, sans aucune affectivité, mais jalouses, envieuses et cruelles. D'après Mustafa elles souffrent de phobies sexuelles, de masochisme, de tabous sexuels excessifs. Une étude faite au Soudan par Abu-el-Futuh ${ }^{5}$ sur 4024 femmes a révélé que plus de $80 \%$ de celles qui avaient subi l'infibulation n'avaient jamais éprouvé l'orgasme. Certaines ignoraient que la femme pût éprouver une quelconque satisfaction lors des rapports sexuels. \& Tout cela a pour résultat une soumission de la femme et sa complète domination par l'homme $»$, conclut Mustafa ${ }^{\circ}$.

\section{Mutilations sexuelles: clitoridectomie rituelle}

C'est à un autre groupe de mutilations sexuelles qu'appartient la clitoridectomie rituelle, telle qu'elle est pratiquée aujourd'hui chez de nombreuses populations africaines noires, en tant qu'élément important des rites d'initiation collective à l'âge de la puberté. Ici aussi, on constate des variations locales, non seulement d'une tribu à l'autre, mais parfois aussi d'un clan à l'autre

4. Cité par El Sayed Mirghani, loc. cit., p. 80-91.

5. Cité par El Sayed Mirghani, loc. cit., p. 111.

6. Cité par El Sayed Mirghani, loc. cit., p. 80-91. 
dans une même tribu. La clitoridectomie peut être simple, partielle ou élargie. Dans tous les cas, il s'agit d'un des actes les plus essentiels d'un rite considéré, là où il existe, comme un des éléments les plus fondamentaux de la vie de la tribu.

Dans l'état encore imparfait de la documentation existante, il serait prématuré d'entreprendre une revue générale qui engloberait toutes les populations chez lesquelles cette coutume existe. Nous nous limiterons à la description de la clitoridectomie rituelle pratiquée dans la tribu des Kikuyu, au Kenya, parce qu'elle est exceptionnellement bien connue au point de vue ethnographique, et qu'elle a donné lieu à des polémiques passionnées dans lesquelles un chef d'État lui-même a jeté le poids de son influence.

Grâce surtout à L.S.B. Leaky ${ }^{7}$ les rites de la circoncision nous sont connus dans le plus grand détail. Nous savons que l'initiation marque la fin de l'enfance et l'admission comme membre de la tribu. Nul ne pourrait se marier s'il n'a pas accompli sa période d'initiation couronnée par la circoncision, tant pour les filles que pour les garçons. Ces rites sont conduits parallèlement, d'un côté pour les garçons, de l'autre pour les filles. Dans la description donnée par Leakey, nous notons que l'opération est pratiquée par une personne hautement spécialisée et dont c'est la profession. Tandis que l'enfant immobilisée par une aide, regarde du côté du mont Kenya, l'opératrice tranche au moyen d'un rasoir le clitoris et une partie des régions adjacentes. L'opération est effectuée sans anesthésie et il importe à la victime de se montrer brave, quoiqu'elle n'y réussisse pas toujours.

Lorsque les Blancs prirent connaissance de ces coutumes, leur attitude fut diverse. Les médecins les condamnèrent formellement tant en raison de la douleur que pour les dangers entraînés : hémorrhagie, infection urinaire, formation de cicatrices épaisses présentant plus tard un danger grave et parfois mortel pour l'accouchement. Les administrateurs coloniaux furent au début fortement opposés à cette pratique et auraient voulu l'abolir, mais ils se rendirent compte qu'elle était trop ancrée pour qu'il fût possible de la supprimer d'un seul coup et ils comptaient sur l'éducation pour en venir à bout avec le temps.

7. L.S.B. Leaky : The Southern Kikuyu before 1903, Academic Press, London, New York, San Francisco, 3 vol. Voir particulièrement vol. 2 , p. 618-624. 
Les missionnaires au début la condamnèrent radicalement et l'interdirent aux nouveaux convertis, espérant que la christianisation finirait par la supprimer. L'opinion publique en Angleterre fut alertée et un groupe de pression se forma en 1930 dans le but d'essayer de persuader le gouvernement colonial de mettre fin à cette coutume barbare. Mais ce fut à un moment où les Kikuyu commençaient à s'agiter pour diverses raisons et à valoriser leurs traditions ancestrales. Une tentative de compromis fut faite : les chrétiens Kikuyu avaient la permission de faire circoncire leurs enfants dans les hôpitaux aseptiquement et sous anesthésie, mais ce compromis fut rejeté, tant par les tenants de la tradition ancestrale que par beaucoup de Kikuyu chrétiens.

A ce moment, L.S.B. Leaky, fils de missionnaire qui connaissait très bien les coutumes des Kikuyu et parlait parfaitement leur langue, intervint dans le débat pour proposer une solution originale ${ }^{8}$. Il rappela que la période d'initiation imposée aux jeunes Kikuyu consistait surtout en l'enseignement des traditions de la tribu, des règles de bonne conduite, l'éducation sexuelle, des chants et des danses. Leaky proposa d'instaurer une nouvelle formule de rites d'initiation. On continuerait à enseigner aux jeunes, au cours d'une sorte de retraite, les règles de bonne conduite, des chants et des danses (en omettant les parties obscènes), en leur donnant également l'instruction sexuelle, et au terme de cette période, on pratiquerait une petite incision sur le bras ou la cuisse, qui serait la marque de la tribu. À mesure que le Kenya se christianisait, cette nouvelle forme d'initiation supplanterait l'autre. Remarquons qu'une coutume semblable existait au Dahomey : les garçons subissaient la circoncision, les filles une incision de forme spécifique sur certaines parties du visage ${ }^{\bullet}$.

Quant aux ethnologues, il semble que leur attitude fut dès l'abord ambiguë. Tout en reconnaissant qu'il y avait des aspects dangereux dans les rites d'initiation, ils insistèrent sur la signification de ces rites et leur fonction de maintenir la cohésion de la tribu. Nous verrons que ces arguments furent repris plus tard par Kenyatta.

8. L.S.B. Leaky : The Kikuyu problem of the initiation of girls. Journal of the Royal Anthropological Society, vol. 6, 1931, p. 277-285.

9. Melville, J. Herskovits, Dahomey, an Ancient West African Kingdom, New York, Augustin, 1938, vol. I, p. 291-299. 
Ceci nous amène à parler de Kenyatta et de son rôle. Nous nous appuierons sur la biographie qu'en a donné Murray-Brown ${ }^{10}$. Kenyatta avait commencé comme « mission boy », c'est-à-dire comme garçon élevé dans une école de missionnaires. Quoique chrétien, il avait subi les rites d'initiation au complet. Plus tard il voyagea en Europe et étudia l'anthropologie à Londres avec Malinowski. De retour au Kenya il se lança dans la politique. Son attitude fut longtemps ambivalente, puis il se dressa ouvertement contre l'autorité coloniale et contre l'enseignement des missionnaires. Après diverses vicissitudes il finit par devenir chef de parti et plus tard chef d'Etat. C'est en 1938 qu'il publia son célèbre ouvrage, Facing Mount Kenya ${ }^{11}$. Ce livre est un chef-d'œuvre d'habileté. Déjà le titre rappelle symboliquement l'attitude des garçons et des filles au moment de la circoncision. Sur la page frontispice, Kenyatta est représenté en costume indigène comme un guerrier Kikuyu, vêtu d'une sorte de couverture, et une sagaie à la main. Il a soin d'indiquer que les Européens ont écorché même le nom de la tribu qui serait non pas celle des Kikuyu mais des Gikuyu (tout en admettant que la vraie prononciation serait Gekoyo). Très habilement aussi, Kenyatta dédicaçait son livre à ses deux enfants Moigoi et Wamboi et à «toute la jeunesse d'Afrique dépossédée : pour la perpétuation de la communion avec les esprits des ancêtres, pour la lutte pour la liberté de l'Afrique, avec la foi inébranlable que les morts, les vivants et ceux qui ne sont pas encore nés se réuniront pour reconstruire les sanctuaires détruits \$. L'ouvrage est présenté comme une monographie ethnographique mais d'un bout à l'autre, c'est une polémique subtile contre les Blancs, les chrétiens et spécialement les missionnaires. Kenyatta affirme que la circoncision, tant celle des filles que celle des garçons, constitue un élément indispensable des rites qui eux-mêmes sont le fondement de la cohésion de la tribu. Cela revient à dire que si l'on supprimait l'aspect chirurgical du rite, tout l'édifice social s'effondrerait. Dans la façon dont Kenyatta expose cet argument, on entrevoit l'influence de son maître Malinowski et de la théorie fonctionnaliste.

10. Jeremy Murray-Brown : Kenyatta, E.P. Dutton \& Co.. Inc., New York, 1973, passim.

11. Jomo Kenyatta : Facing Mount Kenya. The Tribal life of the Gikuyu, Londres : Secker and Warburg Ltd., 1959, p. 130-154. 
Explication de ces coutumes

et des attitudes de la société envers elles

Il est extrêmement difficile de trouver une explication plausible pour l'existence de ces coutumes qui sont très anciennes, puisqu'on les trouve déjà signalées par Hérodote et par Strabon. C'est une notion courante en ethnologie qu'une coutume qui avait sa raison d'être persiste après que le motif originel ait été oublié. Il n'est pas rare alors de lui voir attribuer toutes sortes d'explications d'apparence plausible mais étrangères à son origine véritable, et de voir les masses s'y soumettre aveuglément «parce que la tradition le veut $\gg$.

Dans les populations où ces coutumes règnent aujourd'hui, diverses explications sont offertes aux investigateurs. D'après Corkill, certains croient qu'il s'agit d'une mesure d'hygiène, dont le but serait de protéger la femme contre les vulvo-vaginites. Plus souvent, on entend dire qu'il s'agirait de la protéger contre l'immoralité. Cet argument paraît dérisoire lorsqu'on lit l'enquête faite au Soudan par Abu-el-Futuh ${ }^{12}$, lequel ayant examiné 200 prostituées en trouvait 170 ayant subi l'infibulation, 22 une autre forme de mutilation sexuelles et seulement 8 non circoncises. Certains prétendent que cette opération augmente la fertilité, d'autres au contraire qu'elle la diminue, ce qui équivaudrait à une méthode de contraception. Tiggani ${ }^{13}$ affirme que la circoncision féminine au Soudan est une coutume solide, une tradition religieuse, et qu'elle procure «une heureuse occasion sociale de fêtes joyeuses ».

Une explication par le conflit des générations a été formulée par quelques adeptes de la théorie du matriarcat. Helen Diner ${ }^{14}$ insiste sur le fait que l'opération est pratiquée par des femmes sur d'autres femmes (ce qu'on pourrait interpréter comme un acte d'agression des vieilles femmes sur les femmes très jeunes).

À l'opposé de cette théorie matriarcale, se place la théorie dite sexiste : il s'agit d'un acte d'agression collective des hommes contre les femmes, autrement dit d'une sorte de crime de déféminisation. Déjà Buffon ${ }^{15}$, dans son traité De l'Homme, consacrait

12. Cité par El Sayed Mirghani, loc. cit.

13. Abu-el-Futun : Cité par El Sayed Mirghani, loc. cit.

14. Helen Diner : Mothers and Amazons, New York, The Julian Press, 1965, p. 84-87.

15. Buffon : De l'Homme (réédition), Paris, Maspéro, 1971, p. 89-90. 
une page entière à ces coutumes auxquelles il ne voyait d'autre explication que a la jalousie brutale et criminelle de l'homme envers la femme ».

Il est certain que la circoncision de l'homme n'abolit nullement sa capacité de jouissance sexuelle, tandis que chez la femme son plaisir sera fortement diminué ou aboli complètement suivant le genre d'opération et que dans le cas de l'infibulation la vie sexuelle de la femme sera extrêmement douloureuse.

Il arrive parfois que des hommes ne se contentent pas de donner des explications plus ou moins détournées. Fran Hosken ${ }^{16}$ cite la déclaration d'un homme, en Afrique occidentale, en réponse à une enquête faite par un magazine. Il était certainement en faveur de cette opération disait-il, car, une femme incirconcise est certainement attirée davantage qu'une autre par l'activité sexuelle, et puisque la polygamie était la coutume dans la société à laquelle il appartenait, il lui serait trop difficile de satisfaire toutes ses femmes ; cela ruinerait sa santé. Et s'il ne les satisfaisait pas, ces femmes chercheraient d'autres hommes, ce qui briserait la famille.

L'affirmation de Kenyatta que l'opération est faite avec une extrême rapidité, pratiquement sans douleur et aseptiquement, par une femme "dont l'habileté égale celle d'un chirurgien de Harley Street ${ }^{17}$, ressemble plus à un plaidoyer qu'à une constatation de fait. Quant à l'argument, visiblement inspiré par l'hypothèse fonctionnaliste, à savoir que la circoncision serait le clé de voûte de tout l'édifice social, elle fait penser involontairement à la célèbre apologie du bourreau faite jadis par Joseph de Maistre : sans la présence de cet homme qui fait horreur à tous, tout l'édifice social s'écroulerait. Fort heureusement, la structure d'un édifice social a quand même plus de solidité et de souplesse, et d'ailleurs on connaît des exemples de populations où les rites de circoncision ont été abolis sans entraîner l'effondrement, comme ce fut le cas chez les Zoulous lorsque leur grand chef Shaka abolit cette coutume. D'autre part, l'idée que les générations passées, présentes et futures d'une ethnie constitueraient une sorte de bloc monolithique est un mythe pur et simple, qui ignore l'existence des mutations culturelles (même en dehors de toute influence

16. Fran P. Hosken : Female Circumcision in Africa, Victimology, vol. 2 , $1977-78$, p. $487-498$.

17. Kenyatta, loc. cit., p. 146. 
colonisatrice). Malheureusement, il en résulte que les campagnes pour l'abolition de ces coutumes se heurtent non seulement à la résistance des traditionnalistes, mais encore à ce mythe nouvellement créé.

En réalité, il nous semble que l'infibulisation, ainsi que les autres mutilations infligées aux femmes, représentent un extrême degré d'agression collective de l'homme contre la femme. On y reconnaît un processus de victimogénèse sociale comparable aux suttees des Hindous, à la psychose des sorcières dans l'Europe du $\mathrm{XvI}^{\mathrm{e}}$ et du XVIr ${ }^{\mathrm{e}}$ siècles, et à certains aspects du latah en Indonésie.

C'est ce qui explique peut-être aussi pourquoi les campagnes pour l'abolition de cette coutume se heurtent à de telles résistances en des milieux que l'on aurait cru plus éclairés. A notre connaissance l'Égypte est le seul pays où elle ait été interdite par la loi (1952). Au Soudan et en Somalie, ces opérations sont pratiquées de plus en plus souvent dans les hôpitaux sous anesthésie et aseptiquement, ce qui diminue sans doute les complications, mais ne résout pas le problème. Pire encore, l'Organisation mondiale de la santé, sollicitée à plusieurs reprises de faire une enquête sur cette question, a refusé en alléguant qu'il s'agissait d'un problème social et culturel qui n'était pas de son ressort.

\section{Les mutilations des pieds des Chinoises}

Nous abordons ici une autre coutume à laquelle les auteurs s'accordent à attribuer un caractère érotique, bien qu'elle ne porte pas atteinte aux organes sexuels directement. Il s'agit de la mutilation des pieds des Chinoises. Nous suivrons surtout ici la monographie de S. Howard Lévy ${ }^{18}$.

D'après un auteur chinois du Xrre siècle, cette coutume aurait pris naissance sous le règne de $\mathrm{Li}$ Yü (961-975). Cet empereur, fortement épris d'une concubine très belle, fit construire pour elle un Lotus d'Or, au centre duquel elle déployait ses talents de danseuse. La danse sur un Lotus d'Or était une tradition déjà existante ; elle remontait, semble-t-il, à 4 ou 5 siècles antérieurement. Mais le Lotus d'Or prit une signification nouvelle, celle d'une danse accomplie par une ballerine se distinguant par de très petits pieds bandés. Aux environs de l'an 1 130, la coutume des

18. Howard S. Levy : Chinese Footbinding. The History of a Curious Erotic Custom, New York, Walton Rawls, 1966. 
pieds bandés, originellement limitée à quelques danseuses du harem impérial, commença à se répandre, d'abord chez les autres femmes du harem impérial, puis parmi les femmes de la noblesse. En même temps, la compression devenait de plus en plus forte, et c'était à qui aurait les pieds les plus petits. Les petits pieds bandés devinrent ainsi une marque de distinction entre la noblesse et le peuple, et aussi dans les classes supérieures, un moyen d'assujettissement des femmes aux hommes.

Sous Ie règne des Mongols, cette coutume se diffusa largement à travers toute la Chine. Il est assez curieux que Marco Polo n'en parle pas. En revanche, sous la dynastie des Ming, poètes et romanciers célébrèrent la beauté des petits pieds de femmes. Au $\mathrm{Xvr}^{\mathrm{e}}$ siècle, un roman érotique, Chin $\mathrm{P}^{\prime}$ ing $\mathrm{Mei}$, en parle largement. La mode des petits pieds bandés fut adoptée par quelques dames mandchoues et coréennes, voire même par quelques musulmanes. Ce n'est que sous la dynastie mandchoue que se manifestèrent les premières tentatives pour l'abolition de cette coutume, et les missionnaires chrétiens firent les premiers efforts systématiques dans cette direction.

Ce sont les médecins européens qui exercèrent en Chine au XIX ${ }^{e}$ siècle qui nous ont laissé les descriptions les plus précises sur cette coutume. D'après Morache ${ }^{10}$ il existait deux grands procédés. Dans l'un, les quatre derniers orteils étaient fléchis sous la plante des pieds, le gros orteil laissé libre, le calcaneum restait horizontal. Dans le second procédé, plus radical, la flexion des orteils était plus forte, le calcaneum devenait vertical, de nombreuses lésions se produisaient dans l'articulation tarsienne. On commençait à exercer la compression parfois dès l'âge de 4 ans, au plus tard 6 ou 7 ans. Après des massages du pied, on fixait les bandages en chiffre de huit. Le bandage était changé chaque jour, le pied laissé nu un instant était lavé et frictionné pour prévenir les ulcérations. Parfois la compression était renforcée par l'apposition de pièces de métal sous la face plantaire du pied bandé. Le pied était ensuite chaussé d'une bottine de forme et de taille appropriées. Il fallait continuer à exercer ce genre de compression pendant toute la vie, de peur de voir le pied reprendre peu à peu sa forme normale.

19. G. Morache : Pékin et ses habitants. Etudes d'Hygiène, Annales d'Hygiène Publique et de Médecine Légale, Vol. 22-23, 1869-1870 (Sur la déformation des pieds, voir vol. 32, p. 302-312). 
Il est évident que cette mutilation lente était fort douloureuse. Dans ses souvenirs d'enfance, un Chinois ${ }^{20}$ raconte comment cette coutume se pratiquait aux environs de 1840. Il avait deux jeunes cousines qui vivaient dans la même maison que lui. On commença à leur bander les pieds lorsqu'elles avaient 6 et 8 ans. Il les entendait souvent crier de douleur pendant qu'on mettait les bandages, mais cela n'empêchait pas que pour rien au monde elles n'auraient accepté d'en être dispensées. Cela les aurait rabaissées au rang des servantes. Cette pratique rendait la marche très douloureuse, si bien que les Chinoises ne pouvaient se déplacer que pour de courtes distances.

La compression des pieds entraînait des désordres physiopathologiques de tout genre. La marche était difficile, douloureuse, d'où prédisposition aux chutes, aux entorses, aux fractures de jambe, aux atrophies musculaires et osseuses du membre inférieur. Les troubles atteignaient l'articulation coxofémorale. Les Chinois assuraient qu'il en résultait aussi une hypertrophie des organes génitaux externes.

Du point de vue psychologique, l'effet essentiel consistait dans l'érotisation du pied, sur laquelle ont insisté les médecins européens. La beauté d'une Chinoise était proportionnelle à la petitesse de son pied. Beaucoup d'auteurs affirment qu'en règle générale le sentiment de la pudeur, chez la Chinoise, était localisé dans ses pieds. Dans la peinture chinoise, jamais on ne représentait le pied d'une femme ; toujours la robe le cachait. Regarder les pieds d'une femme passant dans la rue était d'une suprême inconvenance, et personne n'aurait osé parler à un Chinois du pied de sa femme. Pour un mari chinois, disait Matignon ${ }^{21}$, «le pied est plus intéressant que la figure », même si d'après certains rapports les pieds bandés dégageaient parfois une odeur terrible. Le médecin appelé à soigner les pieds d'une Chinoise, avait à surmonter sa pudibonderie. Cependant l'érotisation exerçait ses effets sur l'homme. Il s'agissait d'un véritable fétichisme collectif institutionnalisé. Le Chinois prenait amoureusement dans sa main le pied, le caressait de façon raffinée. "L'attouchement des organes génitaux par le pied produit chez le mâle des frissons d'une

20. Yan Phou Lee : When I was a boy in China, Lothrop, Lowe and Shephard, Boston, s.d., p. 46-49.

21. J.J. Matignon : Superstition, crime et misère en Chine, Lyon et Paris, 2e éd. 1900, p. 311-325. 
volupté indescriptible », écrivait Matignon, et c'est ainsi que les souliers qui avaient chaussé ces petits pieds déterminaient le même effet érotique. « La vue et le toucher de souliers petits et coquets sont l'une des jouissances de ceux auxquels la nature affaiblie refuse d'autres plaisirs \#, écrivait Morache. C'est ce qui amenait parfois ces hommes à boire de l'eau dans l'un de ces petits souliers. Un autre procédé consistait à regarder des albums contenant des images de pieds de femmes - une forme spécifique de pornographie. Les femmes des classes supérieures menaient ainsi une vie de martyres pour être maintenues dans un état d'infériorité physique et pour procurer aux hommes des satisfactions érotiques. Peut-être quelques-unes trouvaient-elles une compensation dans le fait de voir leurs petits pieds chantés par les poètes, plus heureuses en cela que les femmes qui avaient subi des mutilations sexuelles.

Ce qui fait l'intérêt principal des déformations des pieds auxquelles des millions de Chinoises ont été assujetties pendant presque un millénaire, c'est que nous connaissons l'origine historique de cette coutume et ses transformations au cours des siècles jusqu'à son abolition au début du $\mathrm{XX}^{\mathrm{e}}$ siècle. On voit donc ici comment une coutume peut persister et rester impérative à travers les siècles, tout en prenant des significations différentes d'une époque à l'autre et en ayant une pluralité de significations au cours d'une même époque. 